\title{
An aberrant cytoplasmic intron retention programme is a blueprint for ALS-related RBP mislocalization
}

Giulia E. Tyzack $k^{1,2, *}$, Jacob Neeves ${ }^{1,2, *}$, Pierre Klein ${ }^{1,2}$, Hamish Crerar ${ }^{1,2}$, Oliver Ziff ${ }^{1,2}$, Doaa M. Taha ${ }^{1,2,3}$, Raphaëlle Luisier ${ }^{4, \#}$, Nicholas M Luscombe ${ }^{1,5,6 \#, ~ R i c k i e ~ P a t a n i ~}{ }^{1,2, \#}$

${ }^{1}$ The Francis Crick Institute, 1 Midland Road, London NW1 1AT, UK; ${ }^{2}$ Department of Molecular Neuroscience, UCL Institute of Neurology, Queen Square, London, UK; ${ }^{3}$ Zoology Department, Faculty of Science, Alexandria University, Alexandria 21511, Egypt

${ }^{4}$ Idiap Research Institute, Martigny, Switzerland; ${ }^{5} \mathrm{UCL}$ Genetics Institute, University College London, Gower Street, London WC1E 6BT, UK; ${ }^{6}$ Okinawa Institute of Science \& Technology Graduate University, Okinawa 904-0495, Japan; *These authors contributed equally to this work. \#These authors contributed equally to this work.

Key words: Cytoplasmic intron retention, human stem cell model, nucleus, cytoplasm, amyotrophic lateral sclerosis, protein mislocalisation

Correspondence should be addressed to Raphaëlle Luisier (raphaelle.luisier@idiap.ch) and Rickie Patani (ickie.patani@ucl.ac.uk). 


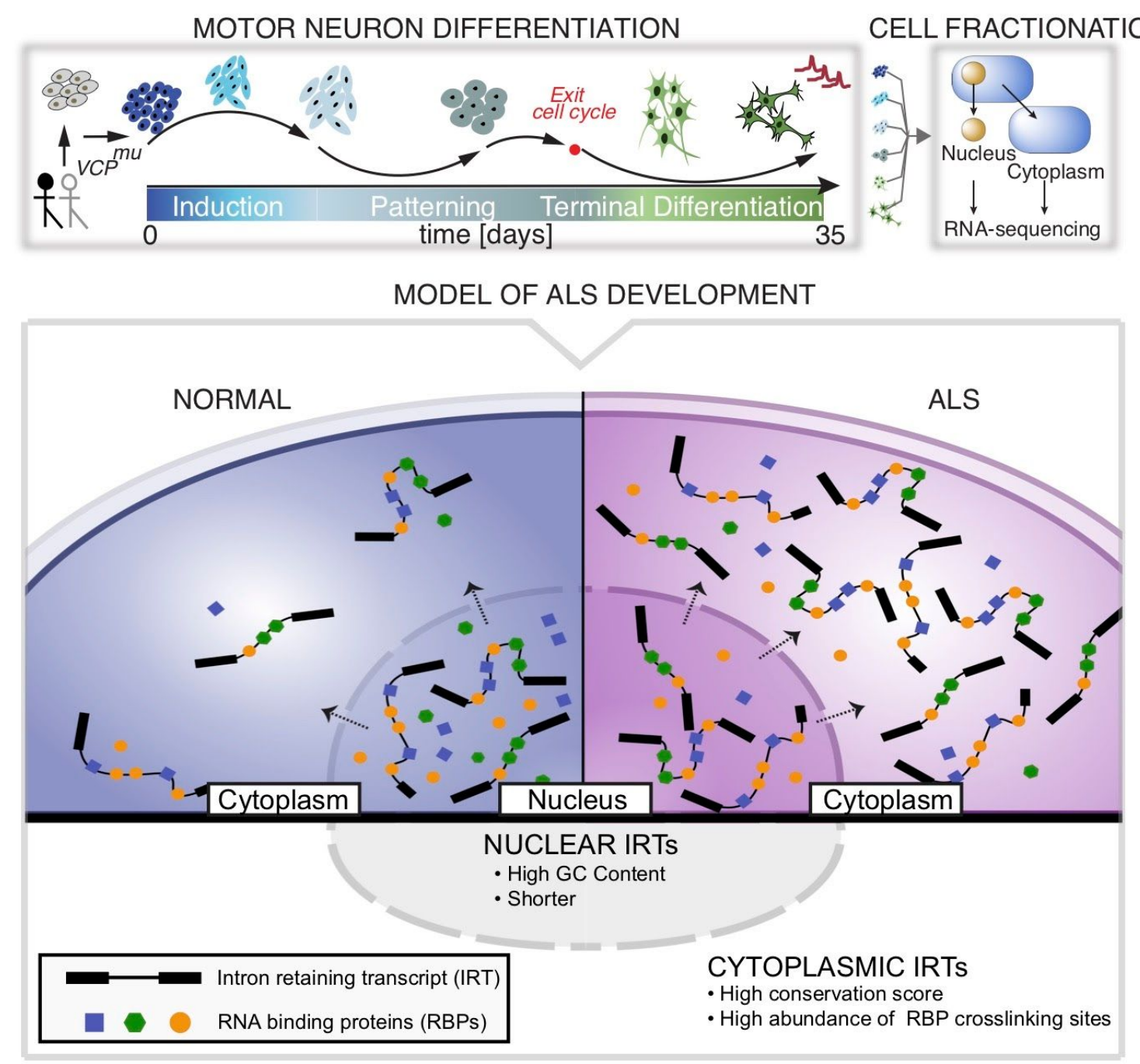




\section{SUMMARY}

We recently described aberrant cytoplasmic SFPQ intron-retaining transcripts (IRTs) and concurrent SFPQ protein mislocalization as a new hallmark of amyotrophic lateral sclerosis (ALS). However the generalizability and potential roles of cytoplasmic IRTs in health and disease remain unclear. Here, using time-resolved deep-sequencing of nuclear and cytoplasmic fractions of hiPSCs undergoing motor neurogenesis, we reveal that ALS-causing VCP gene mutations lead to compartment-specific aberrant accumulation of IRTs. Specifically, we identify $>100$ IRTs with increased cytoplasmic (but not nuclear) abundance in ALS samples. Furthermore, these aberrant cytoplasmic IRTs possess sequence-specific attributes and differential predicted binding affinity to RNA binding proteins (RBPs). Remarkably, TDP-43, SFPQ and FUS - RBPs known for nuclear-to-cytoplasmic mislocalization in ALS - avidly and specifically bind to this aberrant cytoplasmic pool of IRTs, as opposed to any individual IRT. Our data are therefore consistent with a novel role for cytoplasmic IRTs in regulating compartment-specific protein abundance. This study provides new molecular insight into potential pathomechanisms underlying ALS and highlights aberrant cytoplasmic IRTs as potential therapeutic targets.

\section{MAIN}

Studies have demonstrated that intron retention (IR) is more frequent in mammals than originally recognized, affecting transcripts from a majority of genes (Yap et al., 2012a, Braunschweig et al., 2014a; Boutz et al., 2015, Mauger et al., 2016a). Indeed, IR is a prominent mode of splicing during early neuronal differentiation (Braunschweig et al., 2014a; Luisier et al., 2018) and plays a functional role in neuronal homeostasis (Buckley et al., 2011a, Yap et al., 2012b, Braunschweig et al., 2014a, Mauger et al., 2016b). IR during neurogenesis has been shown to exhibit a set of features, an "IR code", that can reliably discriminate retained from constitutively spliced introns (Braunschweig et al., 2014a). IR has previously been implicated in regulating the transcriptome by coupling to RNA degradation pathways (Yap et al., 2012a; Colak et al., 2013; Wong 
et al., 2013, Braunschweig et al., 2014a; Kilchert et al., 2015). Although intron-retaining transcripts (IRTs) have predominantly been identified as residing within the nucleus where they are degraded (Yap et al., 2012b, Braunschweig et al., 2014a; Boutz et al., 2015), there is an expanding body of evidence demonstrating the cytoplasmic localisation of stable IRTs (Buckley et al., 2011b; Khaladkar et al., 2013; Sharangdhar et al., 2017; Saini et al., 2019; Price et al., 2020). It is noteworthy that neural cells, with their exceptional polarity and compartmentalisation, exhibit higher degrees of IR compared with other tissues (Yap et al., 2012a, Braunschweig et al., 2014a, Mauger et al., 2016a). However, their prevalence and role remain understudied. One of the few studies focusing on a cytoplasmic IRT showed that a retained intron in the Calm3 transcript determined its dendritic localization (Sharangdhar et al., 2017), thus revealing an addressing (or 'zip-coding') function of cytoplasmic IR. This study raises the possibility of new roles for intronic RNA sequences beyond a nuclear function, and suggests that cytoplasmic IR programmes are relevant to human neurological function and their perturbation, therefore, to disease.

Amyotrophic lateral sclerosis (ALS) is a rapidly progressive and incurable adult-onset condition, which leads to the relatively selective degeneration of motor neurons (MNs). The molecular pathological hallmark of ALS is the nuclear-to-cytoplasmic mislocalization of key RBPs (Neumann et al., 2006; Luisier et al., 2018; Tyzack et al., 2019), although the underlying mechanism remains elusive. ALS-causing gene mutations implicate crucial regulators of RNA-processing, which are normally expressed throughout development (Sreedharan et al., 2008; Vance et al., 2009). This raises the hypothesis that post-transcriptional changes, including those occurring during neurodevelopment, may play a pivotal role in the underlying molecular pathogenesis of ALS. We recently described IR as the predominant splicing event characterizing early stages of motor neuron lineage restriction from human iPSCs, which is perturbed by genetically diverse ALS-causing mutations (Luisier et al., 2018). However, whether this process affects the nuclear or cytoplasmic subcellular compartments similarly remains unresolved. Few studies have 
examined compartment-specific IR in differentiated neurons (Buckley et al., 2011b; Price et al., 2020), and to our knowledge, no study has comprehensively investigated cytoplasmic IR programmes during human motor neurogenesis, nor systematically characterized the effect of an ALS-causing mutation.

Here, we combine cellular fractionation of hiPSCs undergoing motor neurogenesis with deep RNA-sequencing (RNA-seq) of approximately 100 million paired end reads per sample to gain insights into the molecular 'logic' governing IR programmes in healthy and disease states. This is a rich resource for researchers across the disciplines of basic and applied neuroscience, constituting six timepoints during motor neuron neurogenesis for three control and three ALS hiPSCs lines, which have been separated into nuclear and cytoplasmic fractions.. Indeed, this resource allowed us to make important insights into the nature of aberrant IR in a human stem cell model of ALS. Specifically, we provide a taxonomy for aberrant IR based on nucleocytoplasmic distribution, cis attributes and predicted intron binding affinities to major RBPs. Consequently, we have uncovered a novel class of cytoplasmic IRTs that exhibits predictive value for the nuclear-to-cytoplasmic mislocalization of key RBPs, which constitutes a recognized molecular hallmark of ALS.

\section{RESULTS}

High coverage RNA-seq data from nuclear and cytoplasmic fractions during human motor neurogenesis

We analysed high-throughput poly(A) RNA-seq data derived from nuclear and cytoplasmic fractions of human induced pluripotent stem cells (hiPSC; day 0), neural precursors (NPC; day 3 and day 7), 'patterned' precursor motor neurons (ventral spinal cord; pMN; day 14), post-mitotic but electrophysiologically immature motor neurons (MN; day 22), and electrophysiologically mature MNs (mMNs; day 35). The cellular material was derived from two patients with the ALS-causing VCP gene mutation and four healthy controls (Fig. 1A; 95 samples 
from 6 time-points and 3 genotypes; 4 clones from 4 different healthy controls and 3 clones from 2 ALS patients with VCP mutations: R155C and R191Q, hereafter termed $\mathrm{VCP}^{m u}$ ). Cells from each stage of differentiation were characterised as previously reported (Hall et al., 2017). The efficiency of cellular fractionation was assessed both at protein and RNA level. The predominantly nuclear proteins histone H3 and PSPC1 were highly enriched in the nuclear fraction, whereas the cytosolic enzyme GAPDH was mainly detected in the cytoplasm (Fig. 1B). Similarly, the presence of GAPDH intronic RNA was negligible in the cytoplasm, suggesting that leakage of RNA from the nucleus to the cytoplasm due to the fractionation protocol was minimal. Importantly, the efficiency of fractionation was comparable between control and $\mathrm{VCP}^{m u}$ lines (Fig. 1B). Singular value decomposition analysis of 18,834 reliably expressed genes across the 95 samples revealed that developmental stage and cellular fractions were the largest contributors to transcriptome diversity, explaining $41 \%$ and $15 \%$ of the variance, respectively (Figs. 1C,D). Notably the $\mathrm{VCP}^{m u}$ does not affect these processes and samples cluster with their age- and fraction-matched control counterparts (Supplementary Fig. S1A). Unsupervised hierarchical clustering (Spearman rank correlation and complete-linkage clustering) of the 95 samples using 18,834 genes segregated samples by developmental stage rather than genetic background (Supplementary Fig. S1B).

\section{Widespread aberrant cytoplasmic IR in a human stem cell model of ALS}

We previously identified ALS-related aberrant cytoplasmic SFPQ IRT and concurrent protein mislocalization (Luisier et al., 2018). Here we tested whether aberrant cytoplasmic IR is a generalizable transcriptomic phenomenon in ALS. We examined patterns of splicing using the RNA-seq pipeline VAST-TOOLS (Irimia et al., 2014). We identified 791 nuclear (527 included and 264 skipped) and 329 cytoplasmic (204 included and 125 skipped) alternative splicing events that are statistically significantly different between $\mathrm{VCP}^{m u}$ and control samples in at least one time-point (Supplementary Fig. 1G,H). In line with our previous study, 
the majority of inclusion events between $\mathrm{VCP}^{m u}$ and control samples were retained introns (Fig. 1E). The VCP mutation not only leads to increased IR in the nuclear compartment but also in the cytoplasm (Supplementary Figs. S1C-F). We find that these events peak in pMNs (day 14 in vitro) (Fig. 1F) when we observe a coincident decrease in expression of splicing factors (Fig. 1G and Supplementary Fig. S1I); most notable is the 112 aberrant IR events in the cytoplasmic fraction. Collectively, these findings demonstrate that aberrant cytoplasmic IR is a widespread phenomenon in ALS that occurs during motor neurogenesis.

\section{A nucleocytoplasmic taxonomy for aberrant IRTs}

We next manually curated the list of nuclear and cytoplasmic VCP-mutation related aberrant IR events, focusing on pMNs (day 14 in vitro). We identified three categories of introns that are aberrantly retained in $\mathrm{VCP}^{m u}$ : 1) 237 predominantly in the nucleus; 2) 63 in both the nucleus and the cytoplasm; 3) 49 predominantly in the cytoplasm (Fig. 2A, Tables S3-S5). Gene Ontology functional enrichment analysis shows the specific biological association of affected genes, including cell cycle for the predominantly nuclear IRTs and protein localisation for those that are predominantly cytoplasmic (Fig. 2B). We previously identified aberrant IR in SFPQ, FUS and DDX39A transcripts in VCP ${ }^{m u}$ at an early stage of MN development (Luisier et al., 2018), which we validated here by RT-PCR. Importantly these three IR events belong to the third category (Fig. 2C-D; Supplementary Fig. 2A-B). The finding that a large number of aberrantly retained introns, including SFPQ, DDX39A and FUS, are predominantly localized within the cytoplasm suggests nuclear export and/or cytoplasmic stabilisation, further supporting the hypothesis that $\mathrm{VCP}^{m u}$ impacts distinct IRTs and may lead to stereotyped cellular dysfunction as a consequence. 


\section{Molecular characteristics of aberrantly retained introns}

Prior studies have shown that retained introns are on average shorter and more G/C rich(Galante et al., 2004; Sakabe and de Souza, 2007, Braunschweig et al., 2014a). Strikingly, here we find that only the predominantly nuclear aberrantly retained introns exhibit those features. In complete contrast, aberrantly retained introns within the cytoplasm (in other words, those in both nucleus and cytoplasm, and cytoplasm only) are on average longer and have lower GC content (Figs. 2E,F). Furthermore, the predominantly nuclear aberrant IRTs correlate with a cytoplasmic decrease in gene expression of their non-intron-retaining counterparts: this is consistent with prior observations showing that nuclearly-detained IRTs reduce the level of gene expression (Braunschweig et al., 2014a). Conversely, IR transcripts found in the cytoplasm correlate with increased gene expression within the nucleus (Supplementary Fig. 2E). Importantly this suggests that previously reported features (Galante et al., 2004; Sakabe and de Souza, 2007, Braunschweig et al., 2014a) discriminate nuclearly detained IRTs from cytoplasmic ones. Two additional features further discriminate cytoplasmic-only events from those in both compartments: 1) a high conservation score (Fig. 2G) and 2) a greater abundance of RNA-binding protein (RBP) crosslinking events in the cytoplasmic only retained introns (Sloan et al., 2016; Van Nostrand et al., 2017) (Fig. 2H).

\section{Aberrant cytoplasmic IRTs blueprint ALS-related protein-mislocalization}

The finding that cytoplasmic aberrant IRTs avidly bind to RBPs raises the hypothesis that this interaction drives hallmark RBP mislocalization events in ALS. Indeed we previously reported that the aberrant SFPQ IRT and the SFPQ protein itself, which are predicted to avidly bind to each other, are both exported to the cytoplasm thus providing a potential mechanism for SFPQ mislocalization in ALS (Luisier et al., 2018). We therefore further examined the nature of interaction between RBPs and aberrant cytoplasmic IRTs using our richer dataset. At least 27 RBPs systematically exhibit statistically significant 
increased binding to cytoplasmic-only retained introns compared with their predominantly nuclear counterparts (Fig. 3A). These RBPs form a densely connected network of experimentally validated interacting proteins that are enriched in mRNA metabolism functions (Fig. 3B). The network includes a subset of 9 RBPs with known functions in processing capped intron-containing pre-mRNA, which further implicates disrupted post-transcriptional splicing in ALS pathogenesis. Importantly, also within this network of RBPs are those that exhibit hallmark nuclear-to-cytoplasmic mislocalization in ALS: TDP-43 (Fig. 3B), SFPQ (Fig. 3C) and FUS (Supplementary Fig. 2F) (Neumann et al., 2006; Luisier et al., 2018; Tyzack et al., 2019). Taken together, these findings support the hypothesis that ALS leads to an increase in the cytoplasmic abundance of a class of RNA with a large capacity for binding proteins, thus creating an environment that might encourage mislocalization of their cognate RBPs (Fig. 3D).

\section{DISCUSSION}

Recent studies have demonstrated that IR is more frequent in mammals than previously recognized (Yap et al., 2012a, Braunschweig et al., 2014a; Boutz et al., 2015, Mauger et al., 2016a). It is however noteworthy that the majority of studies have focused on nuclear IRTs and that the importance of cytoplasmic IRTs remains relatively understudied, particularly in the contexts of neuronal development and disease. We previously identified aberrant IR in the SFPQ transcript across human stem cell models of diverse genetic forms of ALS (including those caused by mutations in VCP, SOD1 and FUS genes) (Luisier et al., 2018). In the present study, we sought to understand the generalizability of cytoplasmically localised aberrant IRTs in ALS by combining directed differentiation of patient-specific hiPSCs into spinal MNs, with cellular fractionation and by deep (polyA) RNA sequencing. We showed that aberrant cytoplasmic IR is indeed a widespread molecular phenomenon in ALS that comprises at least 112 transcripts including SFPQ. To better understand the 
nature of cytoplasmic IRTs, we categorized the aberrant IR events into three classes according to their nucleocytoplasmic distribution (nuclear, cytoplasmic and those present in both compartments) and examined their cis attributes.

Retained introns during neurogenesis have previously been shown to exhibit a highly correlated set of cis features comprising an "IR code" that can reliably discriminate retained from constitutively spliced introns (Braunschweig et al., 2014a). Notably the aberrantly retained introns that are predominantly in the nucleus of VCP mutant cultures display similar lengths and GC content to this 'IR code'(Braunschweig et al., 2014a). IR has been previously implicated in fine-tuning the cellular transcriptome by targeting transcripts to RNA degradation pathways such as nonsense mediated decay (Yap et al., 2012a; Colak et al., 2013; Wong et al., 2013, Braunschweig et al., 2014a; Kilchert et al., 2015). The retained introns characterised in the aforementioned studies act broadly to reduce the levels of transcripts that are not required (Boutz et al., 2015; Braun et al., 2017). Indeed only this category of aberrant IR correlates with reduced gene expression in our data. Remarkably, the two other categories we identified exhibit an almost opposite effect on their gene expression levels and thus stimulated further investigation. Our study further revealed a specific class of cytoplasmic IR transcripts that 1) have unique features compared to those reported in previous studies and 2) have conspicuously high affinity for RBPs, including those that are mislocalized in ALS (TDP43, FUS and SFPQ)(Neumann et al., 2006; Luisier et al., 2018; Tyzack et al., 2019). These findings raise the hypothesis that a subset of cytoplasmic IRTs have a distinct role compared to the previously reported IRTs that regulate gene expression and translation through coupling with nonsense mediated decay (NMD)(Braunschweig et al., 2014a). Indeed RNA localization to distinct subcellular compartments has been shown to regulate spatio-temporal control of protein expression(Holt and Bullock, 2009) but less is known about their role in protein localisation. Here we propose that a subset of IRTs aberrantly accumulate in the cytoplasm and their intronic sequences serve as 'blueprints' for the hallmark protein mislocalization events in ALS by creating a mislocalization-prone environment for their bound 
(and usually predominantly nuclear) RBPs (Fig. 3E). This is reinforced by the fact that the RBPs with the largest difference in binding affinity between the predominantly cytoplasmic vs predominantly nuclear aberrant IR are those known to be mislocalized in ALS: TDP-43, FUS and SFPQ. Future studies will directly assess this hypothesis. In summary, we propose that cytoplasmic retained introns function as RNA regulators in the homeostatic control of RBP localisation and that an ALS-related aberrant increase in cytoplasmic IR transcripts disrupts this process. 


\section{FIGURES AND TABLES}

A

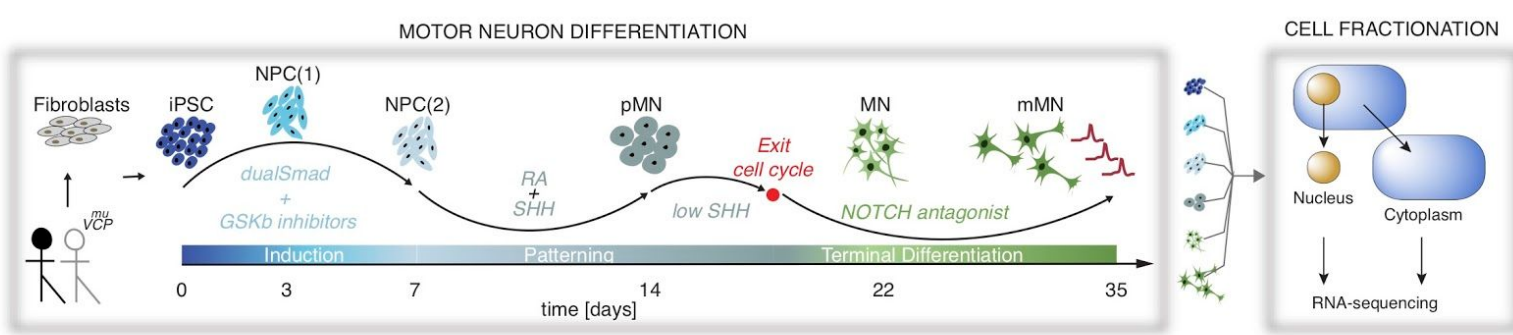

B

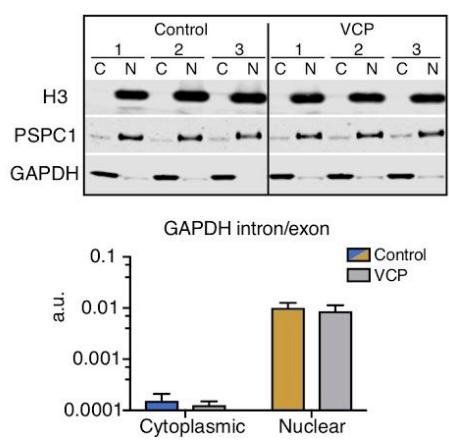

E

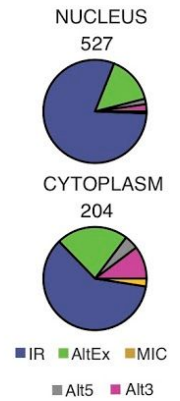

C

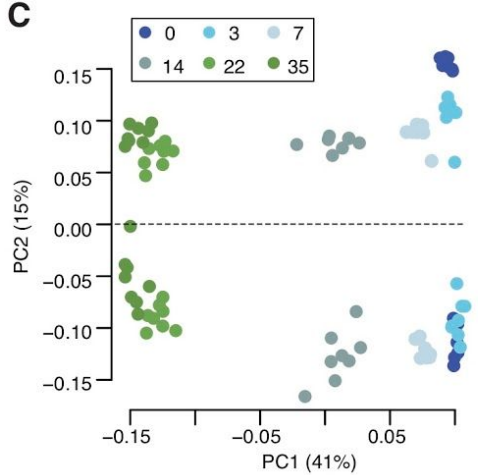

$\mathbf{F}$
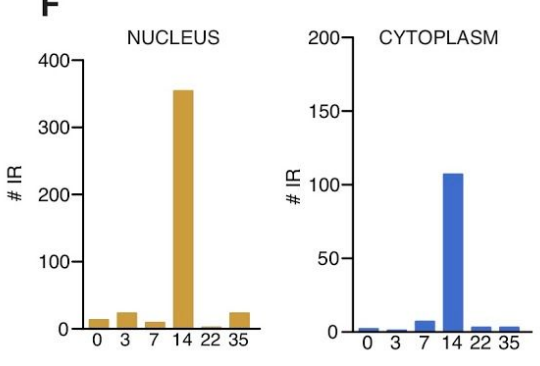

D

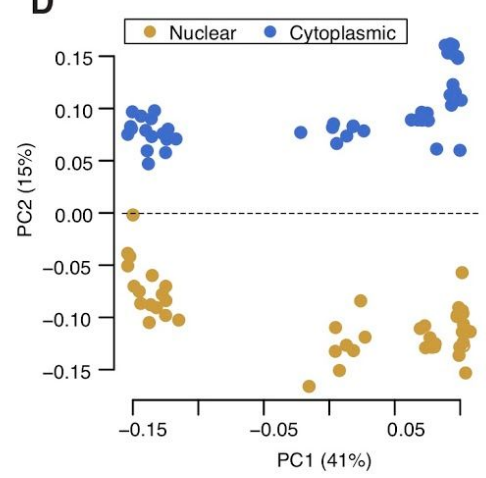

G

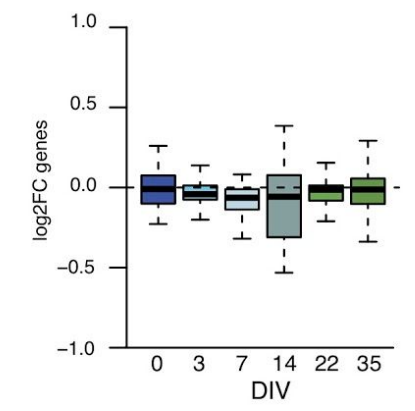

Figure 1 | Time-resolved cellular fractionation and RNA-sequencing during human motor neurogenesis reveals widespread aberrant cytoplasmic IR in ALS. A. Schematic depicting the iPSC differentiation strategy for motor neurogenesis. Arrows indicate sampling time-points in days when cells were fractionated into nuclear and cytoplasmic compartments prior to deep (polyA) RNA-sequencing. Four iPSC clones were obtained from four different healthy controls and three iPSC clones from two ALS patients with VCP mutations: $\mathrm{R} 155 \mathrm{C}$ and R191Q; hereafter termed $\mathrm{VCP}^{m u}$. Induced-pluripotent stem cells (iPSC); neural precursors (NPC); "patterned" precursor motor neurons (ventral spinal cord; pMN); post-mitotic but electrophysiologically inactive motor 
neurons (MN); electrophysiologically active MNs (mMN). B. Representative QC data for fractionation of samples at DIV=14 at protein level (Western blot, top) and RNA level (qPCR, bottom). In the Western blot, histone H3 and PSPC1 were chosen as protein markers for the nuclear faction, and GAPDH was used as cytosolic marker. In the qPCR, the ratio between intronic and exonic GAPDH sequences was measured in both fractions to exclude the leakage of nuclear RNA into the cytosolic fraction due to disruption of nuclei during the fractionation. Data is expressed as mean \pm SD from four lines per group. C. SVD performed on normalized 18,834 gene expression values across 95 samples. Samples are plotted by their coordinates along PC1 (41\% of variance) and PC2 (15\% of variance). Colors of data points indicate similar time in culture: iPSC (dark blue), DIV=3 (blue; NPC1), DIV=7 (light blue; NPC2), DIV=14 (grey; pMN), DIV=22 (lightgreen; MN) and DIV=35 (dark green; eMN). D. Same as (C) with colors of data points indicating similar cellular fractions: nuclear fraction (gold) and cytoplasmic fraction (blue). E. Pie charts representing proportions of included splicing events in $\mathrm{VCP}^{m u}$ at all timepoints of motor neurogenesis compared with age-matched control samples in nuclear (upper chart) and cytoplasmic (lower chart) fractions. Total number of events are indicated above the chart. Intron retention (IR); alternative exon (AltEx); microexons (MIC); alternative $5^{\prime}$ and $3^{\prime}$ UTR (Alt5 and Alt3). F. Bar graphs representing the number of retained introns in $\mathrm{VCP}^{m u}$ compared to control samples at specific timepoints during $\mathrm{MN}$ differentiation. Nuclear fraction (gold; left). Cytoplasmic fraction (blue; right). G. Boxplots showing the distributions of cytoplasmic log2 fold-changes for 72 essential splicing factor genes (Table S8) between $\mathrm{VCP}^{m u}$ and controls. 


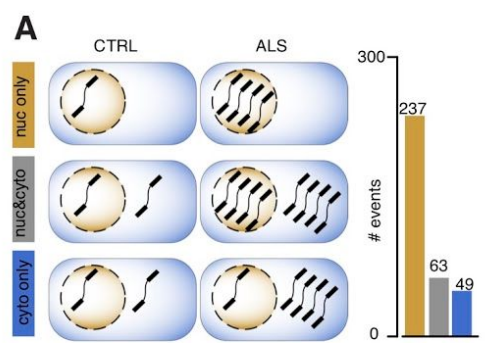

c
B

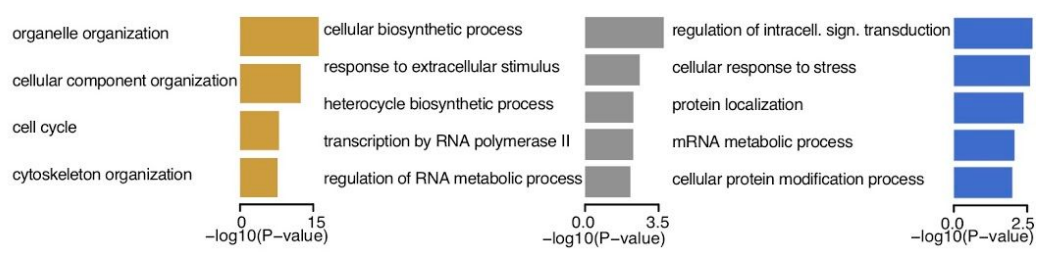

D
NUCLEUS (qPCR)

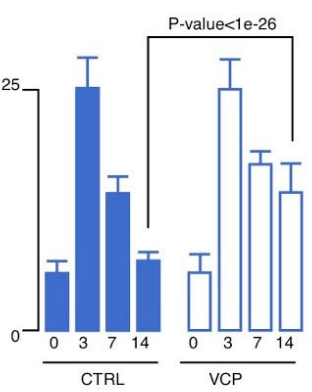

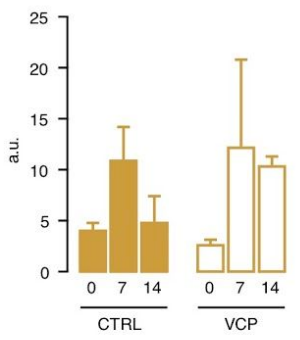

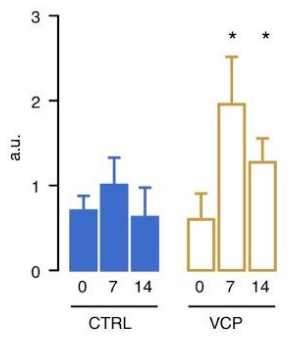

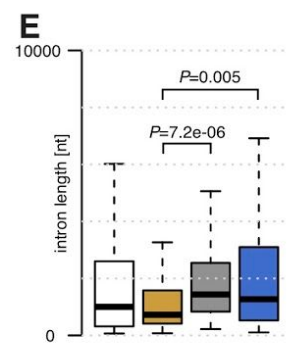

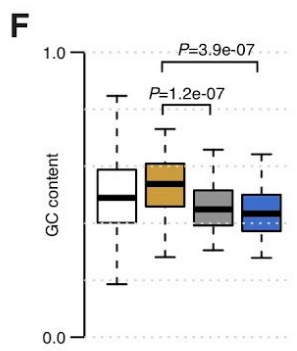

G

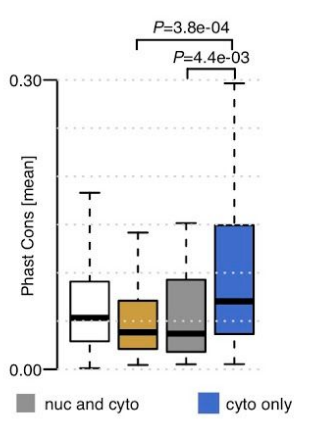

$\mathbf{H}$

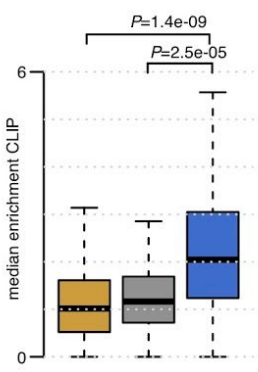

Figure 2 | Aberrant nuclear and cytoplasmic intronic sequences exhibit

distinct characteristics. A. Schematic of our proposed taxonomy for aberrant IRTs (left) and bar graphs (right) representing the numbers of retained introns in $\mathrm{VCP}^{m u}$ compared to control samples at DIV=14 that are predominantly nuclear (gold bar), aberrant in both nucleus and cytoplasm (grey bar), or predominant in cytoplasm (blue bar). The number of events in each category is indicated above the bar. B. Bar plots displaying the enrichment scores for GO biological functions of genes that are targeted by each group of aberrantly retained introns. C. Bar graphs quantifying percentage IR in SFPQ gene at DIV=0, 3, 7 and 14 in control and VCPmu samples (mean \pm s.d.; Fisher count test) in the nucleus (left) and cytoplasm (right). D. Bar plots displaying SFPQ IR levels measured in the nucleus (left) and cytoplasm (right) by qPCR at DIV=0, 7, 14 in control and $\mathrm{VCP}^{m u}$ samples (mean \pm s.d. from four lines per group, ${ }^{*} \mathrm{p}<0.05$, individual $\mathrm{t}$-tests 
comparing control and $\mathrm{VCP}^{m u}$ samples at each time point). E, F, G, H. Comparison of intron length, GC content (\%), conservation scores and median enrichment for RBP binding sites of the three groups of aberrantly retained introns. Data shown as box plots in which the centre line is the median, limits are the interquartile range and whiskers are the minimum and maximum. P-values obtained from Mann-Withney test. All introns in the gene-set targeted by IR in $\mathrm{VCP}^{m u}$ at DIV=14 (white). 
bioRxiv preprint doi: https://doi.org/10.1101/2020.07.20.211557; this version posted July 27, 2020. The copyright holder for this preprint (which was not certified by peer review) is the author/funder, who has granted bioRxiv a license to display the preprint in perpetuity. It is made available under aCC-BY-NC-ND 4.0 International license.

A
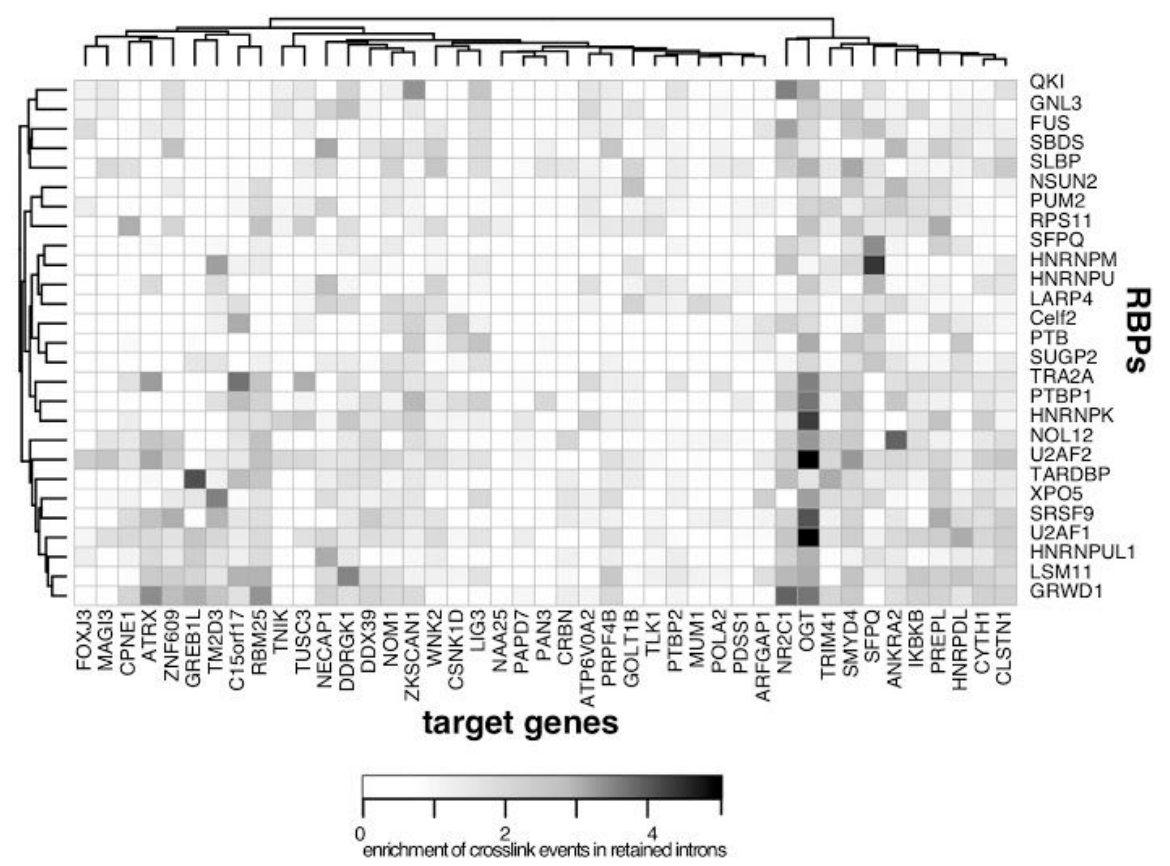

B

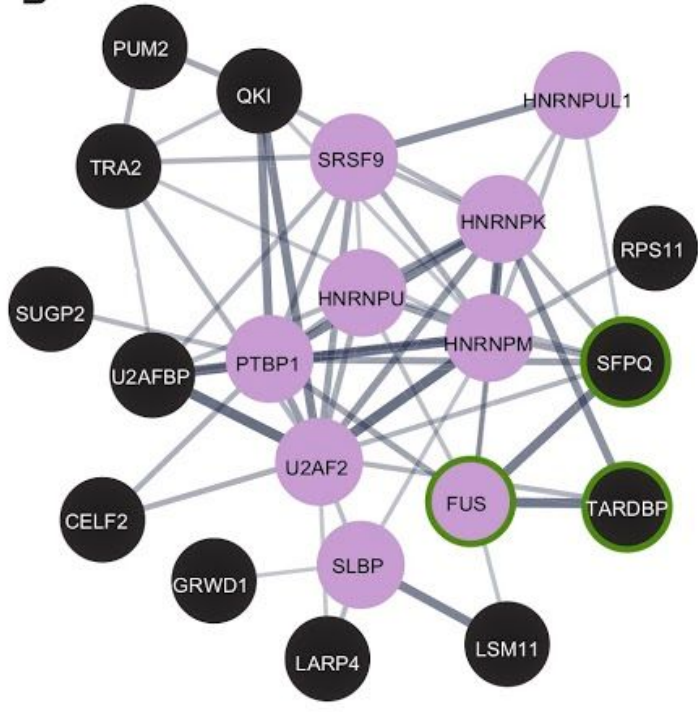

C

D

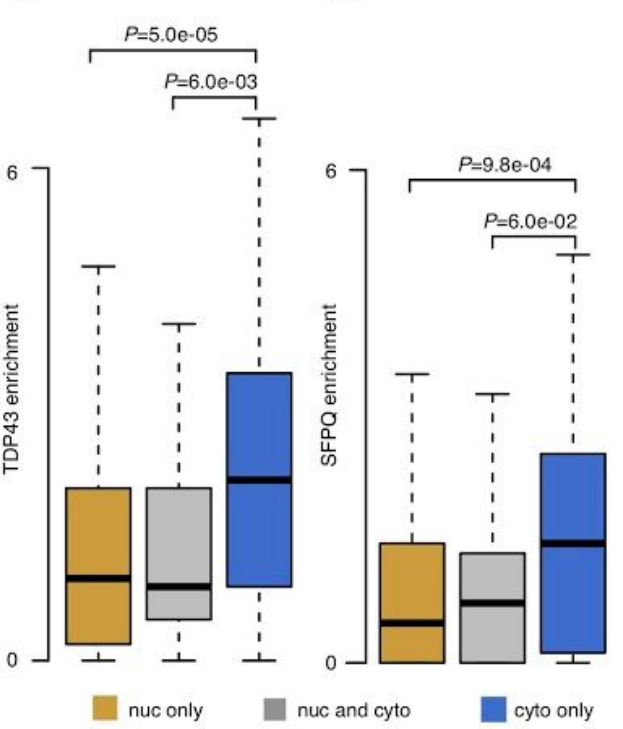

E

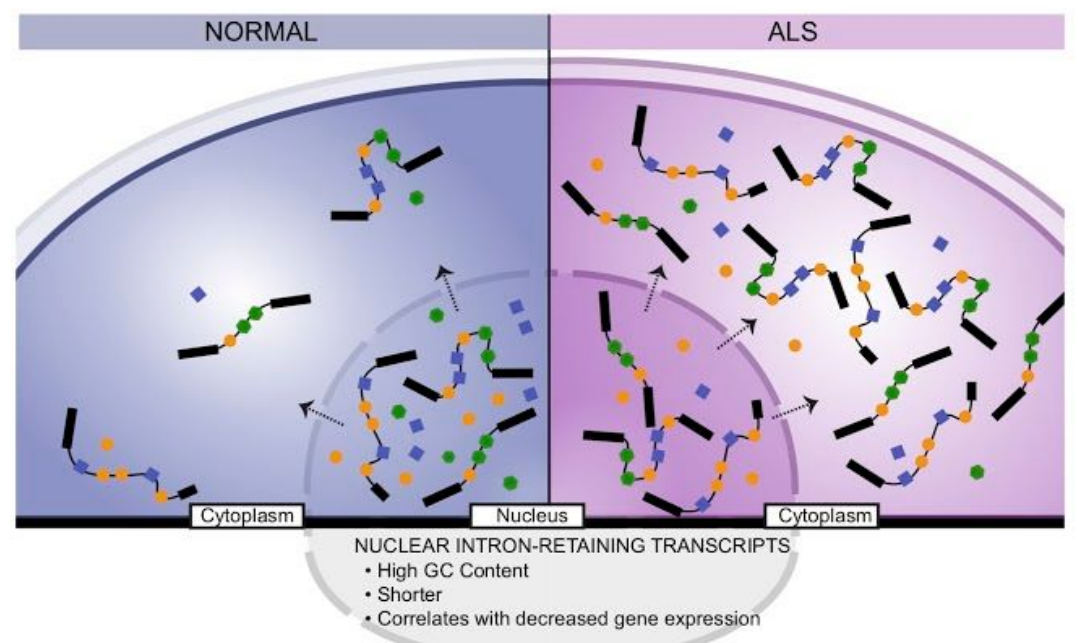

KEY Intron Exon
Extron retaining transcript (IRT)
RNA binding proteins (RBPs)

CYTOPLASMIC INTRON-RETAINING TRANSCRIPTS

- Lower GC Content

- Longer

- Correlates with increased gene expression

- High conservation score

- High abundance of RBP crosslinking sites 
Figure 3 | Cytoplasmic intron-retaining transcripts create a mislocalization-prone environment for bound RBPs. A. Heatmap of the enrichment score of the crosslinking events in each of the 49 predominantly cytoplasmic aberrant IRTs for 27 RBPs that exhibit significantly higher enrichment compared to the two other categories of IRTs (i.e. predominantly nuclear and those that are both cytoplasmic and nuclear). B. Network of protein-protein interactions for 21 (out of the 27) RBPs enriched in cytoplasmic aberrant retained introns. Edges represent experimentally determined protein-protein interactions annotated in the STRING database (Szklarczyk et al., 2017). 9 of these RBPs belong to the Processing of Capped Intron-Containing Pre-mRNA Reactome(Croft et al., 2014) pathway (filled magenta circles) and 3 are RBPs that exhibit hallmark nuclear-to-cytoplasmic mislocalization ALS (green circle). Line thickness indicates the strength of data support based on text mining and experiments. C, D. Comparison of enrichment across all genes within each category for TDP43 and SFPQ crosslinking events in the retained introns between the 3 groups of aberrantly retained introns. Data shown as box plots in which the centre line is the median, limits are the interquartile range and whiskers are the minimum and maximum. P-values obtained from Mann-Withney test. E. Schematic diagram of proposed model where cytoplasmic intron-retaining transcript accumulation in ALS leads to protein mislocalization. 
bioRxiv preprint doi: https://doi.org/10.1101/2020.07.20.211557; this version posted July 27, 2020. The copyright holder for this preprint (which was not certified by peer review) is the author/funder, who has granted bioRxiv a license to display the preprint in perpetuity. It is made available under aCC-BY-NC-ND 4.0 International license.

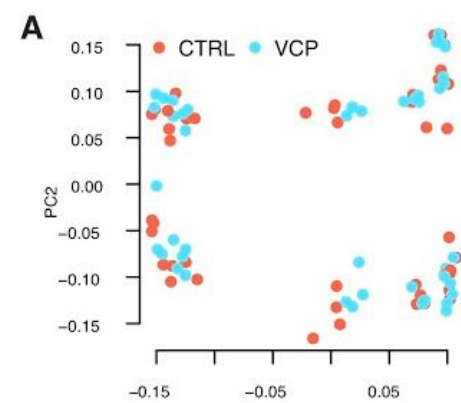

B

iPSC

NPC(1)

$\mathrm{NPC}(2)$

PC1

$\mathrm{pMN}$

MN

$\mathrm{mMN}$

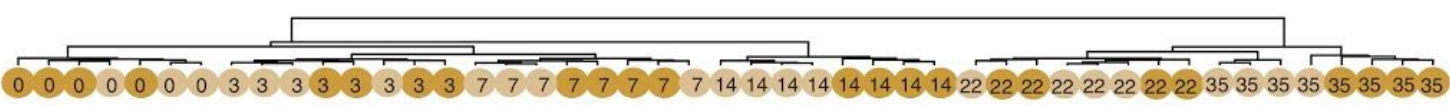

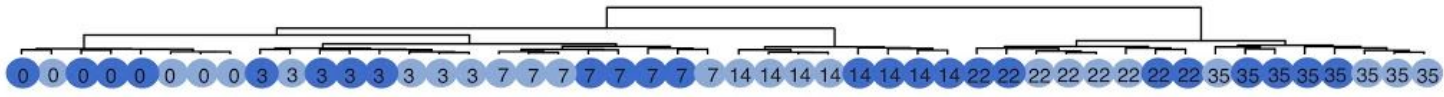

Nucleus CTRL VCP ${ }^{m u}$

Nucleus -

C
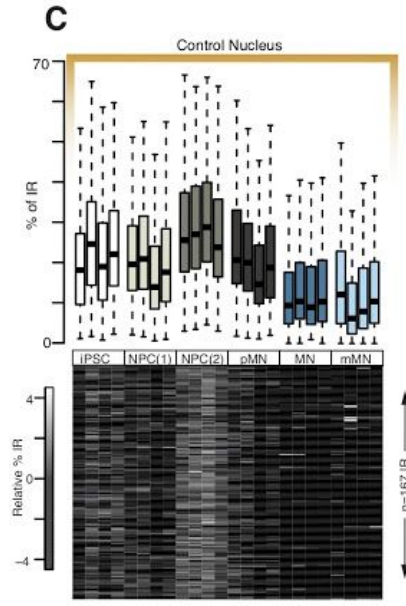

E
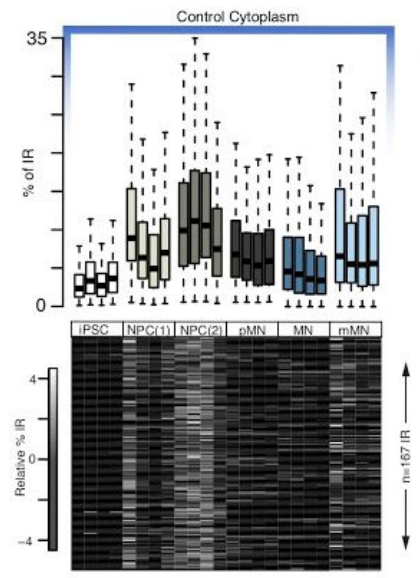

D

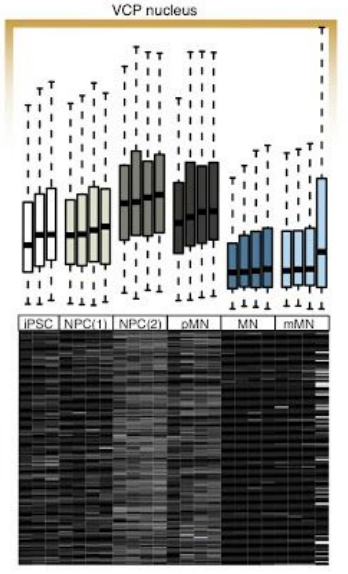

F

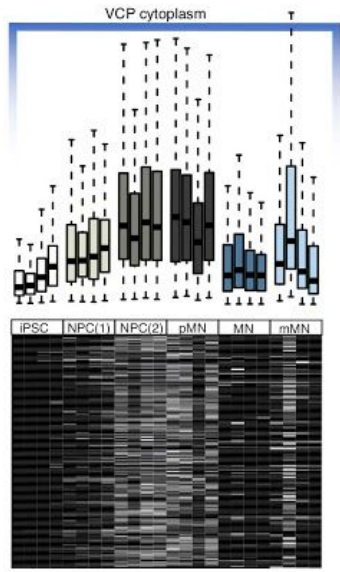

G

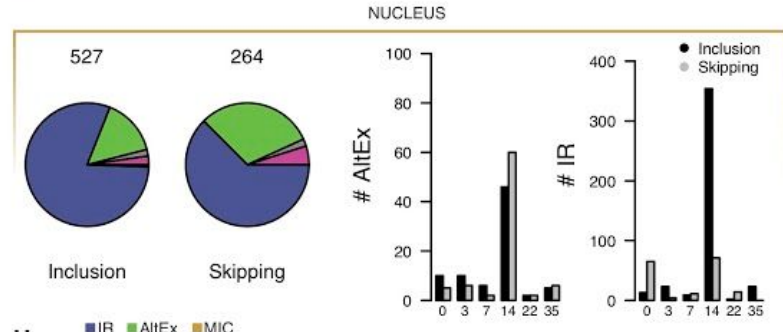

$\mathrm{H} \quad \mathbf{m I R}=\mathrm{AltEX}=\mathrm{MIC}$

mAlt5 $=$ Alt3

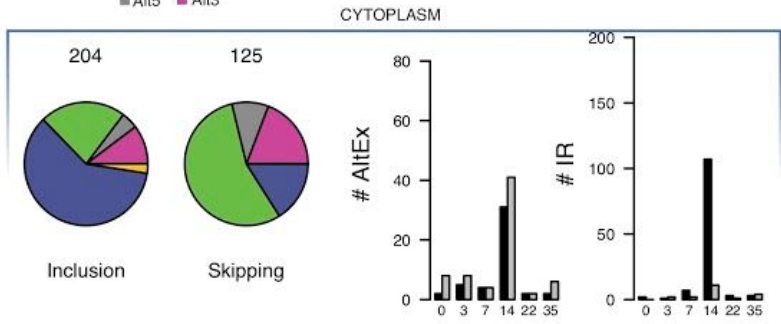

I

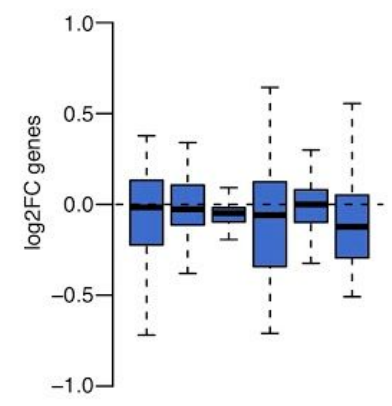


Supplementary Figure 1 | A. SVD performed on normalized 18,834 gene expression values across 95 samples. Samples are plotted by their coordinates along PC1 (41\% of variance) and PC2 (15\% of variance). Colors of data points indicate control or mutated samples: control (red), $\mathrm{VCP}^{m u}$ (magenta). B. Unsupervised hierarchical clustering of 18,834 genes groups the 47 nuclear samples (upper) and 48 cytoplasmic samples (lower) according to developmental stage, rather than genetic background. Dark gold circles = nuclear control samples; light gold circles $=$ nuclear $\mathrm{VCP}^{m u}$ samples; Dark blue circles =cytoplasmic control samples; light blue circles $=$ cytoplasmic $\mathrm{VCP}^{m u}$ samples; sampling time-points are indicated inside the circles. C, D, E, F. Heatmaps of the standardised relative percentage of IR in 167 introns identified in (Luisier et al., 2018) in replicate samples at each differentiation stage in nuclear control samples, nuclear $\operatorname{VCP}^{m u}$ samples, cytoplasmic control samples, cytoplasmic $\mathrm{VCP}^{m u}$ samples respectively. G, H. (left) Pie charts representing proportions of all included and skipped splicing events in $\mathrm{VCP}^{m u}$ at any stages of motor neurogenesis compared with age-matched control samples in nuclear and cytoplasmic fractions respectively. Total numbers of events are indicated above the chart. Intron retention (IR); alternative exon (AltEx); microexons (MIC); alternative $5^{\prime}$ and $3^{\prime}$ UTR (Alt5 and Alt3). (right) Bar graphs representing the numbers of exonic (black bars) and intronic (grey bars) splicing events, in $\mathrm{VCP}^{m u}$ compared to control samples at specific timepoints during MN differentiation. I. Boxplots showing the distributions of nuclear log2 fold-changes for 72 essential splicing factor genes (Table S8) between $\mathrm{VCP}^{m u}$ and controls. Data shown as box plots in which the centre line is the median, limits are the interquartile range and whiskers are the minimum and maximum. 
A

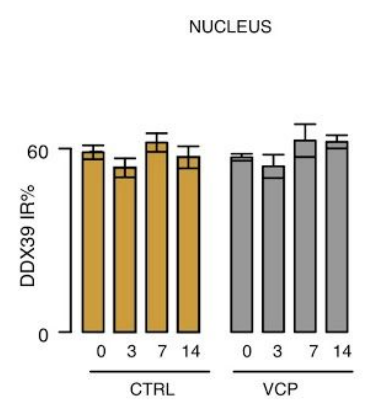

C

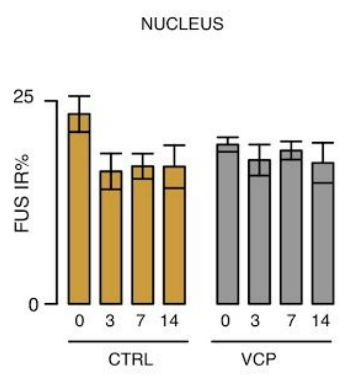

E

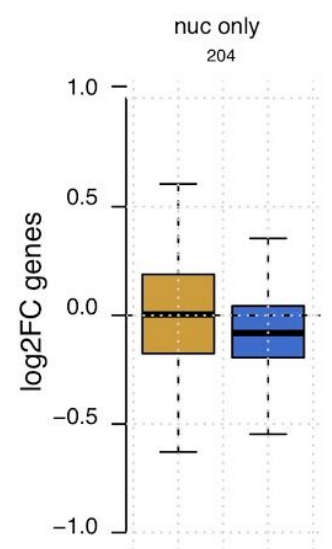

CYTOPLASM

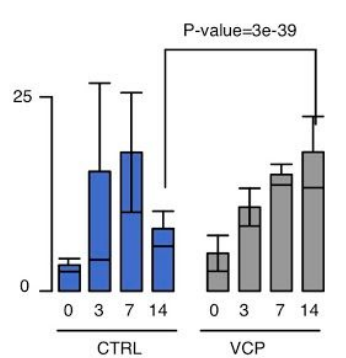

CYTOPLASM

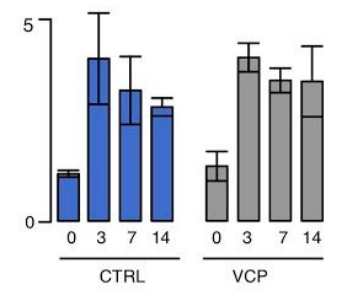

B

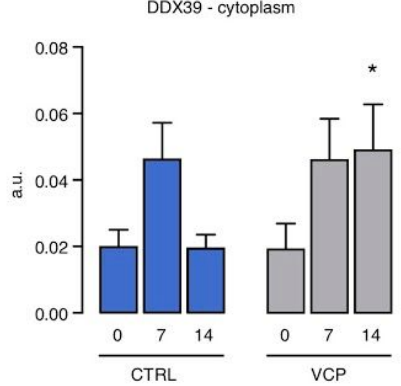

D

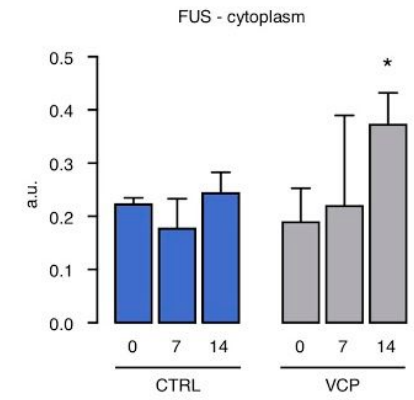

Supplementary Figure 2 | A. Bar graphs quantifying percentage IR in DDX39A gene at DIV=0, 3, 7 and 14 in control and VCPmu samples (mean \pm s.d.; Fisher count test) in the nucleus (left) and cytoplasm (right). B. Bar plots displaying DDX39A IR levels measured in the cytoplasm by qPCR at DIV=0, 7, 14 in control and $\mathrm{VCP}^{m u}$ samples (mean \pm s.d. from 4 lines per group, ${ }^{*} \mathrm{p}<0.05$, individual $\mathrm{t}$-tests 
comparing control and $\mathrm{VCP}^{m u}$ samples at each time point). C. Same as A but for FUS gene. D. Same as B but for FUS gene. E. Boxplots showing the distributions of nuclear (gold) and cytoplasmic (blue) log2 fold-changes for 204, 55 and 43 genes belonging to the three groups of aberrant IR between VCP ${ }^{m u}$ and controls. Data shown as box plots in which the centre line is the median, limits are the interquartile range and whiskers are the minimum and maximum. F. Comparison of enrichment for FUS crosslinking events between the 3 groups of aberrantly retained introns. Predominantly nuclear (gold), nuclear and cytoplasmic (grey), and predominantly cytoplasmic (blue). Data shown as box plots in which the centre line is the median, limits are the interquartile range and whiskers are the minimum and maximum. P-values obtained from Mann-Withney test.

\section{AUTHOR CONTRIBUTIONS}

Conceptualization, R.L., N.M.L., R.P.; Formal Analysis, R.L.; Investigation, R.L., G.E.T., J.N., H.C., P.K., O.Z. D.M.T.; Writing - Original Draft, R.L., R.P.; Writing Review \& Editing, R.L., N.M.L., R.P, G.E.T, J.N.; Resources, R.L., N.M.L., R.P.; Visualization, R.L.; Funding Acquisition, R.L., N.M.L., R.P.; Supervision, R.L, N.M.L., R.P.

\section{ACKNOWLEDGMENTS}

The authors wish to thank the patients for fibroblast donations. We also thank Anob M Chakrabarti for sharing BED files of aligned CLIP data. We thank Mahmoud-Reza Rafiee for useful discussions and advice regarding library preparation for RNA sequencing. We are grateful for the help and support provided by the Scientific Computing section and the DNA Sequencing section of Research Support Division at OIST. This work was supported by the Idiap Research Institute and by the Francis Crick Institute which receives its core funding from Cancer Research UK (FC010110), the UK Medical Research Council (FC010110), and the Wellcome Trust (FC010110). D.M.T. is supported by a Newton-Mosharafa scholarship. R.P. holds an MRC Senior Clinical Fellowship 
[MR/S006591/1]. We also acknowledge Wellcome Trust funding to N.M.L.[103760/Z/14/Z], an MRC eMedLab Medical Bioinformatics Infrastructure Award to N.M.L. (MR/L016311/1). N.M.L. is a Winton Group Leader in recognition of the Winton Charitable Foundation's support towards the establishment of the Francis Crick Institute.

\section{ONLINE METHODS}

\section{Contact for reagent and resource sharing}

Further information and requests for resources and reagents should be directed to and will be fulfilled by the Lead Contact, Rickie Patani

\section{(rickie.patani@ucl.ac.uk).}

\section{Ethics Statement}

Informed consent was obtained from all patients and healthy controls in this study. Experimental protocols were all carried out according to approved regulations and guidelines by UCLH's National Hospital for Neurology and Neurosurgery and UCL's Institute of Neurology joint research ethics committee $(09 / 0272)$.

\section{Cell Culture}

Induced PSCs were maintained on Geltrex (Life Technologies) with Essential 8 Medium media (Life Technologies), and passaged using EDTA (Life Technologies, $0.5 \mathrm{mM})$. All cell cultures were maintained at $37 \mathrm{C}$ and $5 \%$ carbon dioxide. Details of the lines used in this study are provided in Table S1. One of the control lines used (control 3) is commercially available and was purchased fromThermoFisher Scientific (cat. number A18945).

\section{Motor neuron differentiation}

Motor neuron (MN) differentiation was carried out using an adapted version of a previously published protocol (Hall et al., 2017). Briefly, iPSCs were first 
differentiated to neuroepithelium by plating on Geltrex-coated plates to $100 \%$ confluency in chemically defined medium consisting of DMEM/F12 Glutamax, Neurobasal, L-Glutamine, N2 supplement, non essential amino acids, B27 supplement, $\beta$-mercaptoethanol (all from Life Technologies) and insulin (Sigma). Treatment with small molecules from day $0-7$ was as follows: $1 \mu \mathrm{M}$ Dorsomorphin (Millipore), $2 \mu \mathrm{M}$ SB431542 (Tocris Bioscience), and $3.3 \mu \mathrm{M}$ CHIR99021 (Miltenyi Biotec). Starting from day 8, the neuroepithelial layer was patterned for 7 days with $0.5 \mu \mathrm{M}$ retinoic acid and $1 \mu \mathrm{M}$ Purmorphamine. At day 14 pMN precursors were treated with $0.1 \mu \mathrm{M}$ Purmorphamine for a further 4 days before being terminally differentiated in $0.1 \mu \mathrm{M}$ Compound E (Enzo Life Sciences) to promote cell cycle exit. At relevant time points (day 22 and day 35) cells were harvested for cellular fractionation.

\section{Cell fractionation}

Biochemical subcellular fractionation was achieved for all cell stages using the Ambion PARIS kit (ThermoFisher Scientific) cell fractionation buffer, following the manufacturer's general protocol, and an 8M Urea Nuclear Lysis Buffer prepared in house. Initially, cells were washed once using ice-cold PBS. Cytosolic fraction was then obtained by lysing cells directly in ice-cold cell fractionation buffer (ThermoFisher Scientific) for 3-15 minutes, thus disrupting plasma membranes, whilst leaving nuclear membranes intact. Lysates were then centrifuged for 3 minutes at $500 \mathrm{x}$ g at $4 \mathrm{C}$. The supernatant was then collected, further centrifuged at maximum speed in a bench centrifuge at $4 \mathrm{C}$ for 1 minute, and the resulting supernatant was then processed as cytosolic fraction. Nuclear pellets from the first centrifugation step were gently washed once with cell fractionation buffer and then lysed on ice for 30 minutes in $8 \mathrm{M}$ Urea Nuclear Lysis Buffer, containing 50mM Tris-HCL (pH 8), 100mM NaCl, 0.1\% SDS, and $1 \mathrm{mM}$ DTT. The resulting nuclear fraction was then homogenised using a QIAshredder (QIAGEN) to shred chromatin and reduce viscosity, before being further processed for RNA extraction. Both lysis buffers were supplemented 
with $0.1 \mathrm{U} / \mu \mathrm{l}$ RiboLock RNase Inhibitor (ThermoFisher Scientific) and HALT Protease Inhibitor Complex (ThermoFisher Scientific).

\section{RNA extraction and sequencing}

The Promega Maxwell RSC simplyRNA cells kit including DNase treatment, alongside the Maxwell RSC instrument, was used for RNA extractions. For qPCR validations, RNA was extracted using the RNeasy Plus Mini Kit (Qiagen). The nanodrop was used to assess RNA concentration and the 260/280 ratio, and the Agilent TapeStationwas used to assess quality. RNA integrity (RIN) scores were analysed to quality check all samples used in this work.

\section{RNA-sequencing data}

Paired-end polyA stranded RNAseq libraries were prepared from fractionated nucleus and cytoplasm obtained from 6 distinct stages of motor neuron differentiation from control and $\mathrm{VCP}^{m u}$ samples (iPSC, and days 3, 7, 14, 21 and 35) using the NEBNext巴 Ultra ${ }^{\mathrm{TM}}$ II Directional RNA Library Prep Kit for Illumina ${ }$, with NEBNext巴 Poly(A) mRNA Magnetic Isolation Module with 500ng of total RNA as input. Libraries were sequenced at the OIST DNA sequencing section using the NovaSeq 6000 Sequencing technology. 50 bp-long reads were trimmed for adapter sequence and initially aligned to ribosomal RNA sequences to filter out reads that may come from ribosomal RNA contamination using bowtie2 (-v 0) (Langmead and Salzberg, 2012). The remaining reads were aligned to the human genome (hg38) using the splice aware aligner STAR (STAR-2.6.0)(Dobin et al., 2013) with default parameters. All libraries generated in this study had <1\% rRNA, <1\% mtDNA, >90\% strandedness and $>70 \%$ exonic reads (data not shown). One control iPSC nuclear sample failed quality control and was discarded. The list of samples together with the corresponding number of reads in each library and alignment statistics are provided in Table S2. All 
sequence data for this project has been deposited at NCBI GEO database under accession number GSE152983.

\section{Gene expression analysis}

Kallisto (Bray et al., 2016) was used to (1) build a transcript index from the Gencode hg38 release Homo sapiens transcriptome (-k 31), (2) pseudo-align the RNA-seq reads to the transcriptome and (3) quantify transcript abundances (-b 100 -s 50-rf-stranded). Subsequent analysis was performed with the R statistical package version 3.3.1 (2016) and Bioconductor libraries version 3.3 (R Core Team. R: A Language and Environment for Statistical Computing. Vienna, Austria: R Foundation for Statistical Computing; 2013). Kallisto outputs transcript abundance, and thus we calculated the abundance of genes by summing up the estimated raw count of the constituent isoforms to obtain a single value per gene. For a given sample, the histogram of log2 gene count is generally bimodal, with the modes corresponding to non-expressed and expressed genes. Reliably expressed genes for each condition $\left(\mathrm{VCP}^{m u}\right.$ or control at days $0,3,7,14,22$ and 35 in each fraction) were next identified by fitting a two-component Gaussian mixture to the log2 estimated count gene data with $\mathrm{R}$ package mclust (Fraley and Raftery, n.d.) ; a pseudocount of 1 was added before log2 transformation. A gene was considered to be reliably expressed in a given condition if the probability of it belonging to the non-expressed class was under $1 \%$ in each sample belonging to the condition. 18,834 genes were selected based on their detected expression in at least one of the 24 conditions (i.e. 6 different timepoints of lineage restriction for control and $\mathrm{VCP}^{m u}$ in nuclear and cytoplasm). Next we quantile normalized the columns of the gene count matrix with R package limma (Boldstad et al., 2003). Unsupervised hierarchical clustering of the filtered and normalised gene count matrix was performed with Spearman rank correlation as a distance measure and complete clustering algorithm. Principal component analysis has been done with the svd function in R. Differential gene expression analysis was performed with Sleuth comparing VCP mutant or control at each day $(0,3,7,14,22$ and 35$)$ in each fraction. Genes 
that showed a log twofold differential expression and a P-value $<0.05$, and that were reliably expressed in either VCP mutant or control condition were considered as changing significantly.

\section{Splicing analysis}

Intron retention (IR) focussed analysis has been performed on the 167 IR events previously found to be retained during MN differentiation(Luisier et al., 2018) for which a percentage of IR has been calculated as the fraction of intron mapping reads to the average number of reads mapping to the adjacent 5' and 3' exons normalised to the length of the respective intron and exons. A Fisher count test P-value has been obtained when testing for differential IR between conditions. Next the identification of all classes of alternative splicing (AS) events in motor neuron differentiation was performed with the RNA-seq pipeline vast-tools (Irimia et al., 2014). For an AS event to be considered differentially regulated between two conditions, we required a minimum average $\triangle$ PSI (between the paired replicates) of at least 15\% and that the transcript targeted by the splicing event in question to be reliably expressed in all samples from the conditions compared i.e enough read coverage in all samples of interest. We next focussed on the introns aberrantly retained at dat 14 in VCP mutant compared to control samples and conducted Integrative Genomics Viewer (IGV)-guided manual curation to remove low coverage IR obtaining 237 high-confidence IR events that were aberrantly retained predominantly in the nucleus, 63 introns aberrantly retained in both the nucleus and the cytoplasm, and 49 introns predominantly in the cytoplasm. These and their associated characteristics (GC content, conservation score, enrichment in CLIP binding sites) are reported in

\section{Tables S3-S6.}




\section{GO enrichment analysis}

GO enrichment analysis was performed using classic Fisher test with topGO Bioconductor package(Alexa and Rahnenfuhrer, 2016). Only GO terms containing at least 10 annotated genes were considered. A P-value of 0.05 was used as the level of significance. On the figures, top significant GO terms were manually selected by removing redundant GO terms and terms which contain fewer than five significant genes.

\section{Mapping and analysis of CLIP data}

To identify RBPs that bind to aberrantly retained introns, we examined iCLIP data for 21 RBPs(Attig et al., 2018), and eCLIP data from K562 and HepG2 cells for 112 RBPs available from ENCODE (Sloan et al., 2016; Van Nostrand et al., 2017). Before mapping the reads, adapter sequences were removed using Cutadapt v1.9.dev1 and reads shorter than 18 nucleotides were dropped from the analysis. Reads were mapped with STAR v2.4.0i (Dobin et al., 2013) to UCSC hg19/GRCh37 genome assembly. The results were lifted to hg38 using liftOver(Hinrichs et al., 2006) To quantify binding to individual loci, only uniquely mapping reads were used. Relative enrichment for each of the RBPs was obtained by computing the proportion of crosslink events mapping to retained intron compared to non-retained introns of the same genes.

\section{Reverse transcription, $\mathrm{qPCR}$ and intron retention validation}

Reverse transcription was performed using the Revert Aid First Strand cDNA Synthesis Kit (ThermoFisher Scientific) using 500ng-1 $\mu$ g of total RNA and random hexamers. qPCR was performed using the PowerUP SYBR Green Master Mix (ThermoFisher Scientific) and the Agilent Mx3000P QPCR System or the QuantStudio 6 Flex Real-Time PCR System (Applied Biosystems). Primers used are listed in Table S7. Specific amplification was determined by melt curve 
analysis and agarose gel electrophoresis of the PCR products. Primer pairs with 90-110\% efficiency were used. Intron retention validation was performed as previously described (Luisier et al., 2018), and RT-minus samples were used as negative controls. Data was analysed using the $d d C t$ method and is expressed as arbitrary units (a.u. $\left.=2^{-d C t}\right)$.

\section{Electronic supplementary material}

Supplementary Tables 1-8 can be accessed here.

Table S1 | Description of human sample origin and mutations.

Table S2 | Detailed results of the quality control of the 288 fastq files.

Table S3 | List of the 237 IR events that are aberrant at DIV=14 in VCP nuclear compartment predominantly.

Table S4 | List of the 63 IR events that are aberrant at DIV=14 in VCP nuclear and cytoplasmic compartments.

Table S5 | List of the 49 IR events that are aberrant at DIV=14 in VCP cytoplasmic compartment predominantly.

Table S6 | Introns characteristics in terms of length, GC content, maximum entropy at their extremity and conservation scores.

Table S7 | List of primers for qPCR validation of aberrant intron retention.

Table S8 | List of splicing factors used for Figure 1. 


\section{REFERENCES}

Alexa A, Rahnenfuhrer J. topGO: Enrichment Analysis for Gene Ontology. 2016

Attig J, Agostini F, Gooding C, Chakrabarti AM, Singh A, Haberman N, et al. Heteromeric RNP Assembly at LINEs Controls Lineage-Specific RNA Processing. Cell 2018; 174: 1067-1081.e17.

Boldstad BM, Irizarry RA, Astrand M, Speed TP. A Comparison of Normalization Methods for High Density Oligonucleotide Array Data Based on Bias and Variance. Bioinformatics 2003; 19: 185-193.

Boutz PL, Bhutkar A, Sharp PA. Detained introns are a novel, widespread class of post-transcriptionally spliced introns. Genes Dev 2015; 29: 63-80.

Braun CJ, Stanciu M, Boutz PL, Patterson JC, Calligaris D, Higuchi F, et al. Coordinated Splicing of Regulatory Detained Introns within Oncogenic Transcripts Creates an Exploitable Vulnerability in Malignant Glioma. Cancer Cell 2017; 32: 411-426.e11.

Braunschweig U, Barbosa-Morais NL, Pan Q, Nachman EN, Alipanahi B, Gonatopoulos-Pournatzis T, et al. Widespread intron retention in mammals functionally tunes transcriptomes. Genome Res 2014; 24: 1774-1786.

Braunschweig U, Barbosa-Morais NL, Pan Q, Nachman EN, Alipanahi B, Gonatopoulos-Pournatzis T, et al. Widespread intron retention in mammals functionally tunes transcriptomes. Genome Res 2014; 24: 1774-1786.

Bray NL, Pimentel H, Melsted P, Pachter L. Near-optimal probabilistic RNA-seq quantification. Nat Biotechnol 2016; 34: 525-527.

Buckley PT, Lee MT, Sul J-Y, Miyashiro KY, Bell TJ, Fisher SA, et al. Cytoplasmic intron sequence-retaining transcripts can be dendritically targeted via ID element retrotransposons. Neuron 2011; 69: 877-884.

Buckley PT, Lee MT, Sul J-Y, Miyashiro KY, Bell TJ, Fisher SA, et al. Cytoplasmic intron sequence-retaining transcripts can be dendritically targeted via ID element retrotransposons. Neuron 2011; 69: 877-884.

Colak D, Ji S-J, Porse BT, Jaffrey SR. Regulation of axon guidance by compartmentalized nonsense-mediated mRNA decay. Cell 2013; 153: 1252-1265.

Croft D, Mundo AF, Haw R, Milacic M, Weiser J, Wu G, et al. The Reactome pathway knowledgebase. Nucleic Acids Res 2014; 42: D472-7.

Dobin A, Davis CA, Schlesinger F, Drenkow J, Zaleski C, Jha S, et al. STAR: ultrafast universal RNA-seq aligner. Bioinformatics 2013; 29: 15-21.

Fraley C, Raftery AE. mclust Version 4 for R: Normal Mixture Modeling for 
Model-Based Clustering, Classification, and Density Estimation.

Galante PAF, Sakabe NJ, Kirschbaum-Slager N, de Souza SJ. Detection and evaluation of intron retention events in the human transcriptome. RNA 2004; 10 : 757-765.

Hall CE, Yao Z, Choi M, Tyzack GE, Serio A, Luisier R, et al. Progressive Motor Neuron Pathology and the Role of Astrocytes in a Human Stem Cell Model of VCP-Related ALS. Cell Rep 2017; 19: 1739-1749.

Hinrichs AS, Karolchik D, Baertsch R, Barber GP, Bejerano G, Clawson H, et al. The UCSC Genome Browser Database: update 2006. Nucleic Acids Res 2006; 34: D590-8.

Holt CE, Bullock SL. Subcellular mRNA localization in animal cells and why it matters. Science 2009; 326: 1212-1216.

Irimia M, Weatheritt RJ, Ellis JD, Parikshak NN, Gonatopoulos-Pournatzis T, Babor $\mathrm{M}$, et al. A highly conserved program of neuronal microexons is misregulated in autistic brains. Cell 2014; 159: 1511-1523.

Khaladkar M, Buckley PT, Lee MT, Francis C, Eghbal MM, Chuong T, et al. Subcellular RNA sequencing reveals broad presence of cytoplasmic intron-sequence retaining transcripts in mouse and rat neurons. PLoS One 2013; 8: e76194.

Kilchert C, Wittmann S, Passoni M, Shah S, Granneman S, Vasiljeva L. Regulation of mRNA Levels by Decay-Promoting Introns that Recruit the Exosome Specificity Factor Mmi1. Cell Rep 2015; 13: 2504-2515.

Langmead B, Salzberg SL. Fast gapped-read alignment with Bowtie 2. Nat Methods 2012; 9: 357-359.

Luisier R, Tyzack GE, Hall CE, Mitchell JS, Devine H, Taha DM, et al. Intron retention and nuclear loss of SFPQ are molecular hallmarks of ALS. Nat Commun 2018; 9: 2010.

Mauger O, Lemoine F, Scheiffele P. Targeted Intron Retention and Excision for Rapid Gene Regulation in Response to Neuronal Activity. Neuron 2016; 92: 1266-1278.

Mauger O, Lemoine F, Scheiffele P. Targeted Intron Retention and Excision for Rapid Gene Regulation in Response to Neuronal Activity. Neuron 2016; 92: 1266-1278.

Neumann M, Sampathu DM, Kwong LK, Truax AC, Micsenyi MC, Chou TT, et al. Ubiquitinated TDP-43 in frontotemporal lobar degeneration and amyotrophic lateral sclerosis. Science 2006; 314: 130-133.

Price AJ, Hwang T, Tao R, Burke EE, Rajpurohit A, Shin JH, et al. Characterizing the nuclear and cytoplasmic transcriptomes in developing and mature human 
cortex uncovers new insight into psychiatric disease gene regulation. Genome Res 2020; 30: 1-11.

Saini H, Bicknell AA, Eddy SR, Moore MJ. Free circular introns with an unusual branchpoint in neuronal projections [Internet]. Elife 2019; 8Available from: http://dx.doi.org/10.7554/eLife.47809

Sakabe NJ, de Souza SJ. Sequence features responsible for intron retention in human. BMC Genomics 2007; 8: 59.

Sharangdhar T, Sugimoto Y, Heraud-Farlow J, Fernández-Moya SM, Ehses J, de los Mozos IR, et al. A retained intron in the $3^{\prime}$ - UTR of Calm3 mRNA mediates its Staufen2- and activity-dependent localization to neuronal dendrites. EMBO reports 2017; 18: 1762-1774.

Sloan CA, Chan ET, Davidson JM, Malladi VS, Strattan JS, Hitz BC, et al. ENCODE data at the ENCODE portal. Nucleic Acids Res 2016; 44: D726-32.

Sreedharan J, Blair IP, Tripathi VB, Hu X, Vance C, Rogelj B, et al. TDP-43 mutations in familial and sporadic amyotrophic lateral sclerosis. Science 2008; 319: 1668-1672.

Szklarczyk D, Morris JH, Cook H, Kuhn M, Wyder S, Simonovic M, et al. The STRING database in 2017: quality-controlled protein-protein association networks, made broadly accessible. Nucleic Acids Res 2017; 45: D362-D368.

Tyzack GE, Luisier R, Taha DM, Neeves J, Modic M, Mitchell JS, et al. Widespread FUS mislocalization is a molecular hallmark of amyotrophic lateral sclerosis. Brain 2019; 142: 2572-2580.

Vance C, Rogelj B, Hortobagyi T, De Vos KJ, Nishimura AL, Sreedharan J, et al. Mutations in FUS, an RNA Processing Protein, Cause Familial Amyotrophic Lateral Sclerosis Type 6. Science 2009; 323: 1208-1211.

Van Nostrand EL, Freese P, Pratt GA, Wang X, Wei X. A large-scale binding and functional map of human RNA binding proteins [Internet]. bioRxiv 2017Available from: https://www.biorxiv.org/content/early/2017/08/23/179648.abstract

Wong JJ-L, Ritchie W, Ebner OA, Selbach M, Wong JWH, Huang Y, et al. Orchestrated intron retention regulates normal granulocyte differentiation. Cell 2013; 154: 583-595.

Yap K, Lim ZQ, Khandelia P, Friedman B, Makeyev EV. Coordinated regulation of neuronal mRNA steady-state levels through developmentally controlled intron retention. Genes Dev 2012; 26: 1209-1223.

Yap K, Lim ZQ, Khandelia P, Friedman B, Makeyev EV. Coordinated regulation of neuronal mRNA steady-state levels through developmentally controlled intron retention. Genes Dev 2012; 26: 1209-1223. 
bioRxiv preprint doi: https://doi.org/10.1101/2020.07.20.211557; this version posted July 27, 2020. The copyright holder for this preprint (which was not certified by peer review) is the author/funder, who has granted bioRxiv a license to display the preprint in perpetuity. It is made available under aCC-BY-NC-ND 4.0 International license. 